Molina Guixot, Claudia.

Doctoranda en el programa de doctorado en Arte: Producción e Investigación, Universidad

Politécnica de Valencia.

\title{
La Creación Artística Digital: Procesos de Transferencia Inkjet.
}

\section{Digital Art Creation: Inkjet Transfer Processes.}

\author{
TIPO DE TRABAJO:
}

Comunicación.

PALABRAS CLAVE:

Transferencia, inkjet, proceso, gráfica digital.

KEY WORDS:

Transfer, inkjet, process, digital graphic.

\section{RESUMEN.}

En este artículo se reflexiona sobre la construcción de nuevos imaginarios partiendo de la esencia reproducible perteneciente a la mirada tradicional del grabado, que dota de multiplicidad a la imagen, hasta llegar a un nuevo pensamiento: no sólo por las tecnologías de generación e impresión de imágenes digitales, fundamentales hoy en día como herramientas para los artistas, sino también por el nuevo concepto que se tiene de obra múltiple.

Sistemáticamente se centra en el análisis y desarrollo de nuevos procesos de transferencia, así como en la exploración de nuevas posibilidades en el campo de la gráfica digital, mediante impresiones realizadas con sistemas de impresión de inyección de tinta (inkjet) cuyo fin es la creación artística. Estos métodos surgen como respuesta necesaria a las limitaciones de los soportes que pueden ser utilizados en las impresoras inkjet tanto de pequeño como gran formato.

Conceptualmente este procedimiento expone nuevas reflexiones ligadas al recorrido que realiza la tinta de la imagen como materia, atendiendo a cambios y pérdidas de tinta, debido al desplazamiento intencionado que sufre la imagen, hasta ser transferida sobre el soporte definitivo. Estas modificaciones no sólo están ligadas a las variaciones físicas de la imagen, sino también a las transformaciones que el movimiento digital genera sobre las imágenes, sometiéndolas a tratamientos o manipulaciones, cuestionando la idea de origen.

El objetivo principal de esta investigación se centra en la búsqueda de procesos capaces de traspasar imágenes que han sido generadas e impresas con sistemas de impresión inkjet, hacia soportes que debido a su naturaleza no son compatibles con la tecnología de la impresora.

\section{ABSTRACT.}

This article reflects on the construction of new imagery starting from the reproducible essence belonging to the traditional look of engraving, which gives multiplicity to the image, until arriving at a new thought: not only by the technologies of generation and printing of images Digital, fundamental today as tools for artists, but also for the new concept that has multiple works. 
It systematically focuses on the analysis and development of new transfer processes, as well as the exploration of new possibilities in the field of digital graphics, through prints made with inkjet printing systems whose purpose is artistic creation. These methods come as a necessary response to the limitations of media that can be used in both small and large format inkjet printers.

Conceptually this procedure exposes new reflections linked to the path that the ink of the image makes as a matter, taking into account changes and losses of ink, due to the intentional displacement of the image, until it is transferred onto the final support. These modifications are not only linked to the physical variations of the image, but also to the transformations that the digital movement generates over the images, subjecting them to treatments or manipulations, questioning the idea of origin.

The main objective of this research is focused on the search for processes capable of transferring images that have been generated and printed with inkjet printing systems, towards media that due to their nature are not compatible with the technology of the printer.

\section{CONTENIDO}

\section{INTRODUCCIÓN.}

A lo largo de la historia, el grabado ha sido catalogado como un proceso que se encargaba de reproducir obra, mayoritariamente de las técnicas más relevantes como el dibujo o la pintura, con el objetivo de difundirlas. Esta multiplicidad de obras, calificada como copia, desvalorizó al grabado como un arte mayor dentro del ámbito artístico.

Con la aparición de la tecnología digital y, más concretamente con los sistemas de impresión, el campo del grabado y la estampa experimenta un cambio de paradigma. La técnica adopta un nuevo lenguaje autónomo, donde la finalidad de la reproducción de las imágenes queda lejos del nuevo concepto que se tiene de la gráfica, capaz de generar obra original. Se introducen nuevos tecnicismos que se adaptan a la era digital y se cambia la denominación de las obras realizadas con tecnologías electrónicas y programas informáticos. Según José Ramón Alcalá "la gráfica es, ahora, gráfica multimedia, gráfica audiovisual, gráfica interactiva, gráfica en movimiento, gráfica-luz y gráfica-código, gráfica de la red y en la red." (2011, p. 67) ${ }^{1}$. Tomando como premisa estas palabras se construye una mirada que va más allá de una técnica y que adopta el nombre de: gráfica digital.

Bajo este pretexto, el artículo plantea un estudio sobre la ampliación de nuevas posibilidades en el campo de la gráfica digital y las nuevas lecturas que la cultura digital provoca sobre técnicas tan tradicionales como es el grabado. Estas aportaciones vienen dadas, por la introducción, como herramienta fundamental para la creación artística, de los sistemas de impresión de inyección de tinta (inkjet), impresoras presentes en nuestro día a día como un electrodoméstico más.

El interés de la autora por estas máquinas viene dado por las limitaciones que presentan para depositar tinta sobre soportes que, debido a sus cualidades y morfología, no pueden ser impresas. Es por ello que, en esta investigación, se centra en el análisis y desarrollo de nuevos procesos de transferencia como solución a esta restricción.

La transferencia no solo trata de un procedimiento encargado de trasladar imágenes impresas de un lugar a otro; también implica una reflexión procesual que atiende a los cambios o pérdidas de la propia tinta como materia. Durante el recorrido desde que la imagen es impresa sobre un soporte temporal hasta asentarse sobre un soporte definitivo, la imagen se ve involucrada en situaciones que generan nuevos códigos y que, por tanto, afectará a la apariencia final de esa imagen.

Con el desarrollo de procesos de transferencia de tina inkjet, se pretende ampliar los usos de la gráfica, aplicando esta metodología sobre nuevas superficies que vayan más allá del tradicional papel y diluir los límites entre copia y original e, incluso, cuestionarnos la idea de origen.

\section{DESARROLLO.}

\section{- INICIOS DEL TRANSFER Y LOS SITEMAS INKJET.}

Para comprender la necesidad de investigar acerca de procesos de transferencia con la tecnología de impresión inkjet, tenemos que realizar una retrospección a través de los distintos procedimientos existentes, tipos de máquinas o creaciones de artistas, separando, por un lado, el origen de la técnica y, por el otro, el comienzo de los sistemas de inyección de tinta. 
Los inicios históricos del proceso de transferencia, desde sus formas más arcaicas, se registran a finales del siglo XVIII, momento en que se inventaron mecanismos y máquinas con la finalidad de reproducir y copiar topo tipo de documentos. Valgan como ejemplo el método introducido por James Watt o el Mimeógrafo desarrollado por Thomas Alva Edison en el 1875; estos sistemas evolucionaron mejorando la técnica y abriendo nuevos horizontes. Ya en el siglo XX, concretamente en 1940, se introdujo la transferencia por difusión, una técnica que se basaba en dos papeles (positivo y negativo), los cuales se introducían en líquido revelador, pegados por la cara emulsionada; tras unos minutos, se separaban y aparecía la imagen, gracias al efecto del papel preparado químicamente llamado el transergo.

Paulatinamente, el desarrollo de estos sistemas fue incrementándose y especializándose, tanto el proceso en sí, como en los materiales empleados, con el objetivo de alcanzar un equilibrio entre el registro, el medio transportador y el soporte definitivo.

Este proceso cautivó al artista Robert Rauschenberg, el gran referente y antecesor de esta técnica. Este artista exploró y popularizó este procedimiento como recurso plástico, a partir de 1958, cuando transfería con disolvente las imágenes captadas de los media.

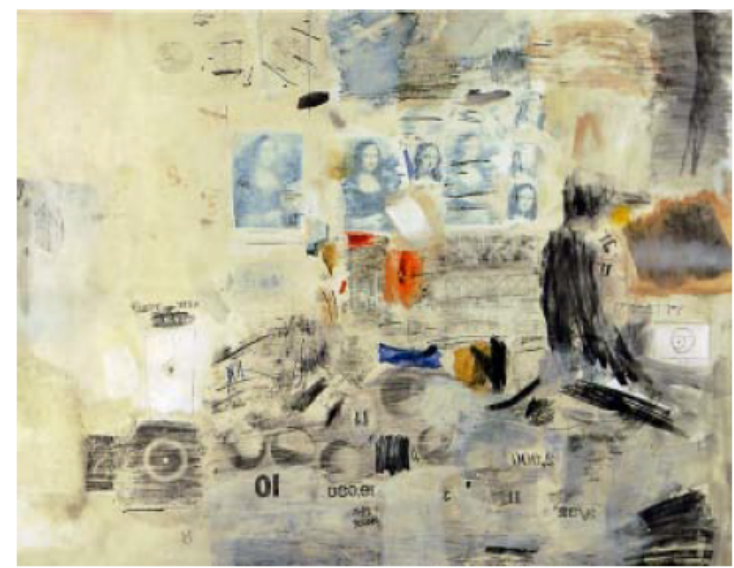

Fig. 1. RAUSCHEMBERG, ROBERT, Courrency serie Combines, transferencia disolvente y acrílico sobre papel, 1958.

En el panorama nacional cabe destacar las aportaciones de Jesús Pastor, quien realizó multitud de estudios sobre grabado en cobre y hierro, partiendo de la transferencia de fotocopias. Otro autor de referencia, Paco Rangel, fue de gran notoriedad en las décadas finales del siglo XX con sus electrotransfers. A su vez, Rubén Tortosa, destaca por sus transferencias sobre soportes poco convencionales como el cristal, cera, óleo, poliuretanos acrílicos y látex.

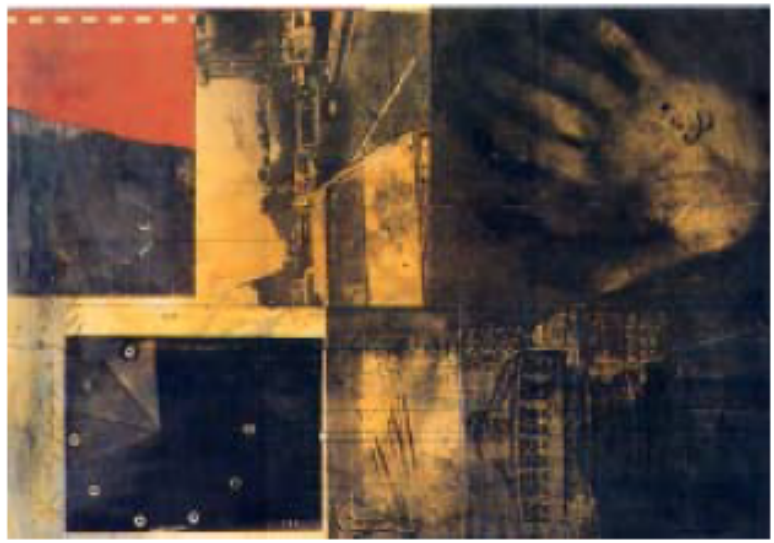

Fig. 2. RANGEL, PACO, Inmortal II, electrotransfer, 1991. 


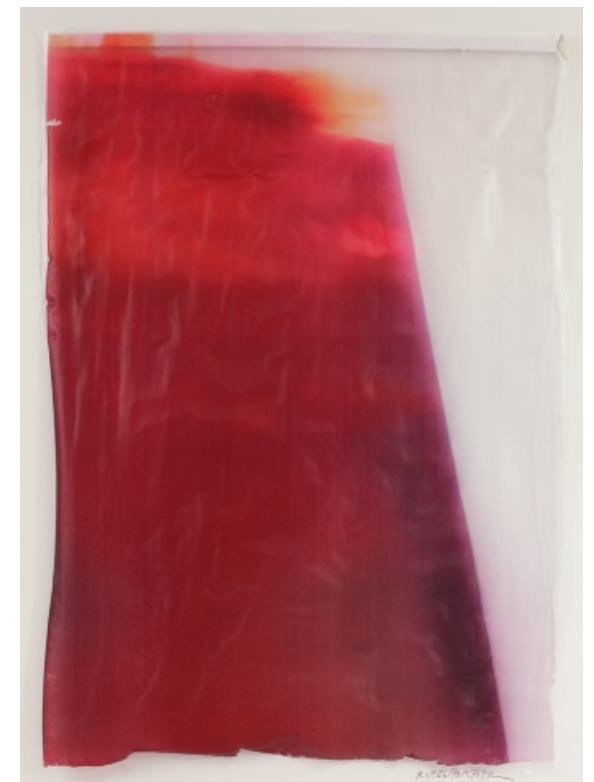

Fig. 3. TORTOSA, RUBÉN, Print, impresión digital transferida sobre poliuretano, 2013.
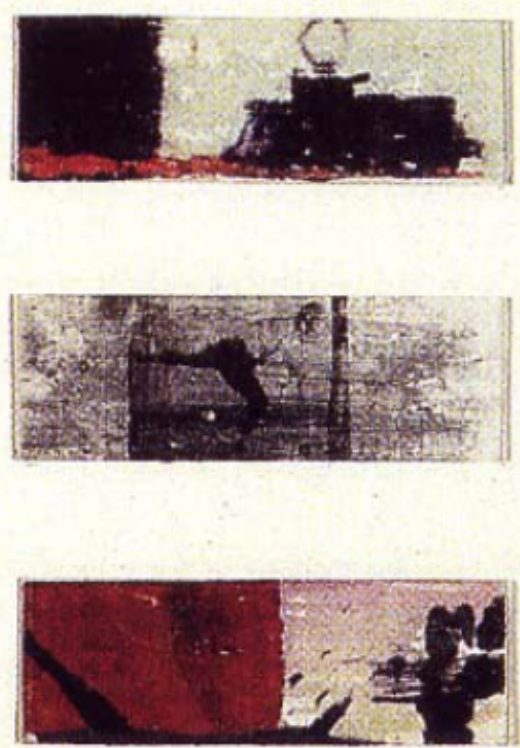

Fig. 4. TORTOSA, RUBÉN, S/T transferencia sobre cristal, 1998.

A comienzos del siglo XX los sistemas de impresión inkjet se fabricaban con fines técnicos, desarrolladas para impresiones de gran formato. Pero fueron muchos los artistas que encontraron en estos sistemas capacidades interesantes para ser aprovechadas y cambiar la visión que se tenía de estas máquinas como reproductoras de imágenes y elevarlas a la categoría de creadora de obras de arte.

Robert Rauschenberg, no sólo es relevante porque introdujo la técnica de la transferencia en sus creaciones, si no, porque, además, fue uno de los pioneros en transferir imágenes emanadas de los sistemas de impresión inkjet, gracias a la naturaleza de la tinta que utilizaba la conocida impresora IRIS. 


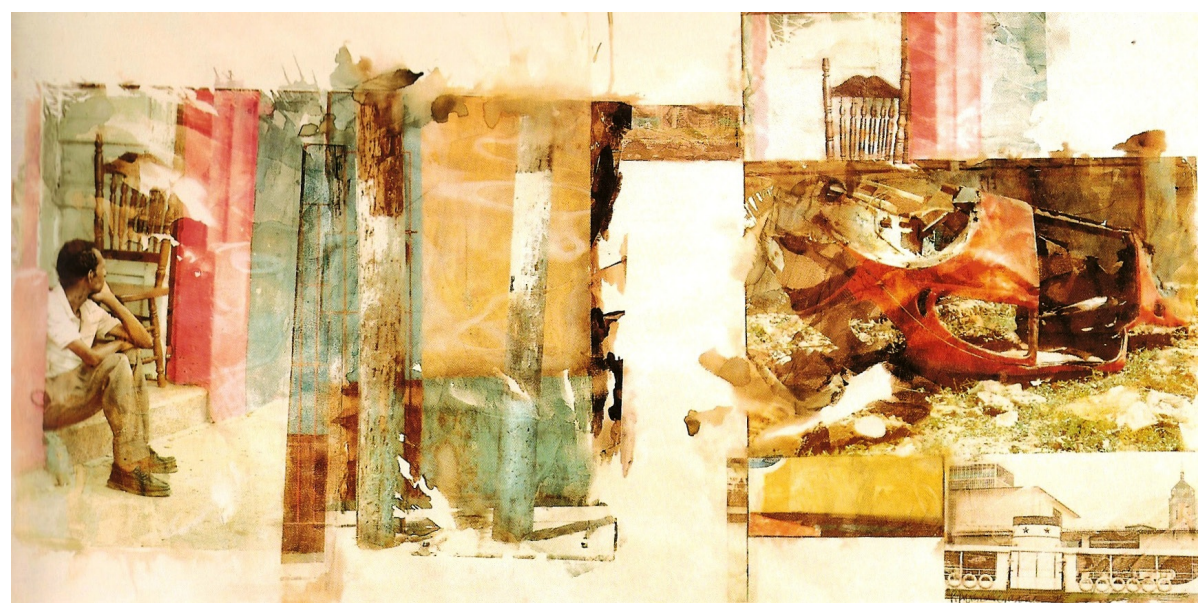

Fig. 5. RAUSCHEMBERG, ROBERT, Chairman (Anagram), transferencia con tinte vegetal sobre papel, 1993.

De hecho, durante los últimos 30 años, han sido muchos los artistas que han utilizado y siguen haciendo uso de las máquinas impresoras de inyección de tinta para la creación de obras de arte sobre papeles de alta calidad o hibridando otros materiales para alcanzar resultados diferentes. Como ejemplo representativo, cabe citar a los hermanos Starn; estos artistas utilizaban como punto de partida en sus obras la impresión inkjet, que combinaban con otros materiales para conseguir negros muy intensos. David Hockney, fotógrafo y pintor entre otras disciplinas, amplió su campo de actuación más allá de la pintura, explorando la realidad con ordenador y las nuevas tecnologías de impresión.

\section{- $\quad$ PROCESOS DE TRANSFERENCIA INKJET.}

En este apartado se especifica la parte técnica de la propuesta, necesaria para entender el conjunto del artículo y el discurso que se pretende transmitir a través del proceso de transferencia de tinta inkjet.

La transferencia o transferir según la RAE (Real Academia Española) significa: pasar o llevar algo desde un lugar a otro. Es fundamental señalar la definición de este término para entender mejor el paralelismo que existe entre el proceso como técnica artística y el proceso como concepto, el cual nos hace reflexionar acerca de esta traslación.

A continuación, se explica y enumera de manera breve los componentes metodológicos necesarios para llevar a cabo el proceso de forma satisfactoria y obtener resultados óptimos. Es importante conocer la temporalización y orden del proceso, al igual que los materiales empleados, para entender lo que ocurre dependiendo de los diferentes niveles por los que pasa la imagen. Estos acontecimientos, que van transformando los códigos de la imagen, pueden producirse tanto en la generación de la propia imagen como en la última de las representaciones sobre el soporte final.

Para explicar adecuadamente el proceso de transferencia inkjet, se necesita de una cierta extensión, ya que además de los componentes que a continuación señalaremos, existen otros elementos básicos para la confección del proceso como, por ejemplo, la composición de las diversas tintas o cómo funcionan los diferentes soportes temporales empleados durante la investigación. Para la realización de este articulo nos centraremos en los materiales y factores más relevantes para poder llevar a cabo el correcto desarrollo.

Inicialmente es necesaria la creación de una imagen mediante sistemas de registro digitales como puedan ser la cámara fotográfica o el escáner, entre otros. Una vez generada, la imagen es sometida a tratamientos o manipulaciones a través de programas informáticos, finalmente se imprime sobre el soporte temporal. Seguidamente se explica de donde procede este concepto y que función realiza en el proceso.

La denominación de soporte temporal se debe a la función que cumple durante la transferencia. Este elemento contiene la imagen que es impresa sobre él, pero solo durante un determinado espacio-tiempo, ya que actúa como intermediario para transportar la imagen hacia la superficie que actuará de soporte definitivo. Existen varios tipos de papeles, pero, para este artículo, nombraremos el 
más notorio de la investigación, a pesar de tratarse de un papel no destinado para este fin, es el que mejor resultados hemos obtenido. Se trata del acetato para inkjet, un papel con un recubrimiento especial, único para este tipo de tintas.

Otro elemento fundamental para el entendimiento y la concepción del cómputo general de la imagen, es el soporte definitivo. Esta superficie necesaria para concluir el proceso de transferencia, es de especial interés para la investigación, pues se trata del material que otorga de un estado corpóreo a la imagen digitalizada y el que le concede de un valor añadido a la obra; es por ello por lo que resulta significativo de analizar y experimentar con el soporte definitivo para encontrar la armonía íntegra entre imagen y dicho soporte.

Pero para alcanzar el equilibrio entre materiales, primero hay que realizar el paso de transferir la imagen convertida en tinta hacia la superficie final, es decir, el medio reportador que nos hará alcanzar nuestro propósito. Teniendo en cuenta la naturaleza de la tinta, existen varios métodos que hacen posible este traslado. Uno de ellos es mediante disolución: se aplica una mezcla de agua y látex que permite que la tinta se desprenda del soporte temporal para asentarse sobre el definitivo, combinándolo con ayuda de la presión.

El segundo de los métodos utilizados para reportar la imagen de un lugar a otro, es mediante la compatibilidad de medios, es decir, el propio soporte actúa a la vez de medio reportador de la tinta. En este caso se trata de látex, ya que, gracias a su cualidad de transformarse de líquido a sólido una vez aplicado sobre el soporte temporal, se consigue transferir la tinta desde este soporte temporal hacia el definitivo (látex) transcurrido unas horas, añadiendo algún otro componente que ayuda a completar con éxito el proceso.

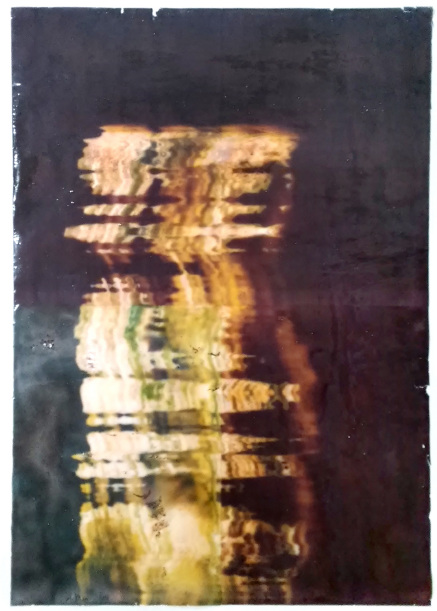

Fig. 6. MOLINA GUIXOT, CLAUDIA, Registro gráfico_digital, transferencia de tinta inkjet sobre látex, 2013.

Finalmente, la imagen queda plasmada sobre el material que hemos elegido acorde al discurso, el cual queremos envolver a la imagen. Además, se pretende dejar patente, no solo la huella de la impresión, sino también la propia huella, es decir, hacer presente la propiedad de lo manual y singular, donde el artista pasa a ser parte integrante y determinante del proceso.

\section{- $\quad$ EL MOVIMIENTO DE LA TRANSFERENCIA.}

Para lograr entender de manera holística el proceso de transferencia, en este apartado se explica el efecto de este transcurso desde una perspectiva más crítica y reflexiva y como, partiendo de la globalización de lo digital, puede convertirse en algo único y original.

La transferencia de tinta inkjet se basa en un desplazamiento que nace con la representación de una imagen, trasmitida a través de un dispositivo que la construye y codifica para, finalmente, mostrarnos una interpretación de una imagen real. Esta imagen versionada y digitalizada se transforma en analógica y en materia (imagen impresa mediante máquinas inkjet), plasmándose sobre el soporte temporal en forma de micro gotas ordenadas para mostrarnos dicha imagen. Finalmente se traspasa hacia el soporte definitivo mediante sistemas de reporte que obligan a la tinta a desprenderse de su asentamiento.

Como hemos observado durante el proceso vamos traspasando la imagen de un nivel a otro, según Bourriaud "cualquier signo ha de ser traducido, o traducible" (2009, p. 153) ${ }^{2}$, desde lo global y lo abstracto, hasta lo local y lo concreto. Este traducir es fundamental para entender la transferencia como un lenguaje que transcribe los códigos que va recorriendo la imagen, pues como señala Kako Castro "necesitamos de máquinas que desvelen el misterio encerrado en los números" $(2004$, p. 32) 3 . Estas máquinas, en nuestro caso 
el escáner, pantalla de ordenador e impresora, van trasladando signos digitales de una imagen considerada como real que, sin embargo, se asemeja, pero que, tras ser digitalizada, produce unos cambios que genera nuevos códigos.

La imagen está sometida a un continuo movimiento: la propia formalidad del proceso le obliga a trasladarse de un lugar a otro, a traducir los códigos que la conforman. En un determinado espacio-tiempo pertenece atrapada en la interfaz; más tarde será extraída en forma de materia. Pero, durante ese periodo, la imagen está sometida a manipulaciones o modificaciones en la que la propia máquina interpreta nuestro pensamiento. Así pues, para poder contener esta imagen tenemos que guardarla o, en este caso, traducirla, a un formato (jpg, tiff, ...) que nos permita su representación a través de la pantalla. Este flujo intangible hace que se disuelva la autenticidad de la imagen, alejándonos cada vez más del origen y la originalidad.

Siguiendo con la formalidad del proceso, la imagen se traslada hacia una superficie física y se transforma en materia, en este caso en tinta inkjet procedente de los sistemas de impresión de inyección de tinta. Aquí la responsabilidad de traducir y transferir la imagen recae sobre la materia. Interpreta los signos digitales en forma de gotas que conforman un aspecto semejante a la imagen codificada por la pantalla. Tal y como apunta Kako Castro "No tenemos ninguna garantía de que al final obtengamos nada que se le parezca" $\left(2004\right.$, p. 34) ${ }^{4}$. Es conveniente subrayar esta premisa, porque no solo dependemos de una máquina, sino también de la versión de la tinta en su conjunción y el soporte donde se dispondrá la misma.

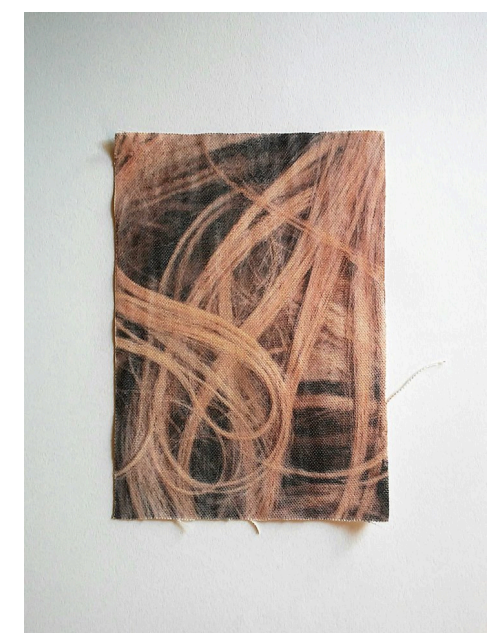

Fig. 7. MOLINA GUIXOT, CLAUDIA, S/T, transferencia de tinta inkjet sobre tela, 2016.

El tipo de tinta ejerce un papel importante y determinante en el proceso de transferencia inkjet, pues es la protagonista de esta metodología. Como se observa, la acción de trasladar sigue impulsada por el desarrollo del proceso, ahora de una forma física y palpable. Este desplazamiento provoca un nomadismo obligado de la tinta, la cual queda presente en cada uno de los asentamientos. Primero es extraída de su depósito, el cartucho, por el calentamiento de unos inyectores, después es liberada sobre la superficie que actúa como soporte temporal y, finalmente es reportada sobre el material que ejerce de soporte definitivo. Como se puede comprobar, el rastro de la tinta queda patente y deja huellas visibles en cada una de las partes que compone la orden de transferir.

Durante este nomadismo, la tinta como materia sufre unos cambios y pérdidas que afectan al aspecto final de la imagen. No solo por las cualidades de la tinta, sino también porque existe un determinado momento en donde la intervención de lo manual se hace visible y cobra protagonismo sobre la obra final. Este aspecto es fundamental tanto a nivel procesual como personal. Ser parte del proceso implica que se derive esa mecanización repetitiva, propia de los sistemas tecnológicos y digitales, y que se haga presente la irrupción humana en el proceso de transferencia.

Finalmente, tras traducir, trasladar y transferir, una similitud de la imagen inicial queda corporizada y aferrada en un material no convencional. 

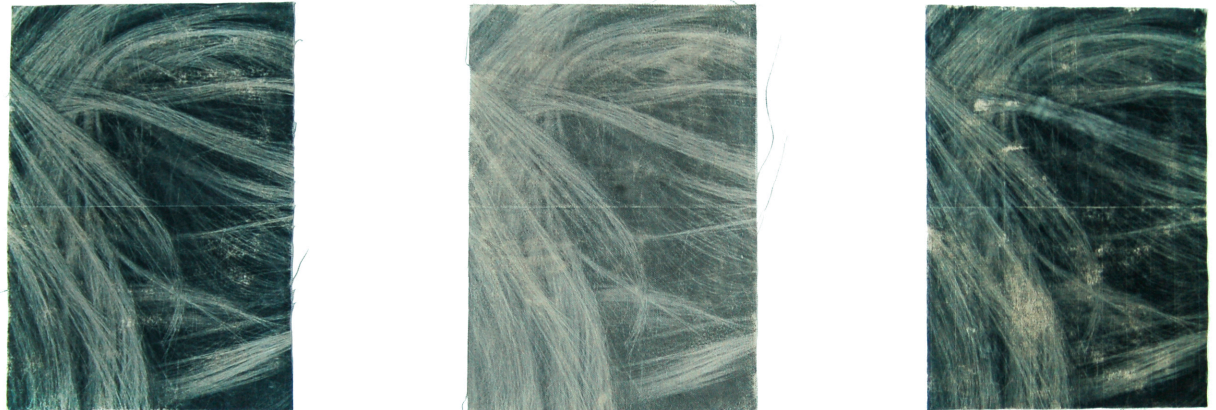

Fig. 8. MOLINA GUIXOT, CLAUDIA, Variaciones físicas, transferencia de tinta inkjet sobre tela, 2016.

\section{CONCLUSIONES.}

Para concluir este artículo, se pretende señalar los aspectos más relevantes que envuelven a la transferencia de tinta inkjet, como un proceso que se engloba dentro del ámbito de la gráfica digital, catalogándolo como un arte actual y flexible que permite modelar multitud de discursos, ampliando el campo de visión de este lenguaje. De la misma forma:

- Destacar al proceso de transferencia de tinta inkjet como una solución válida, para estampar imágenes emanadas de los sistemas de impresión de inyección de tinta, sobre soportes que debido a su morfología no son capaces de ser impresos.

- Desde el punto de vista conceptual, entender que la transferencia, se encarga de traducir los códigos de un formato a otro, desvelando la imagen planteada por una combinación de pixeles y cuestionándonos la idea de origen y originalidad de la misma.

Estas traslaciones son las que hace que repensemos como afecta la globalización de los medios y sistemas digitales en nuestra cultura. Este movimiento es el responsable de determinar a cualquier imagen reproducida por la pantalla como autentica y real, y no como algo engañoso, que solamente es posible retener, si la imagen digital se transforma en un elemento semejante físico y palpable.

\section{FUENTES REFERENCIALES.}

ALCALÁ, JOSÉ RAMÓN, 2010. Ser Digital. Chile: Departamento de Artes Visuales, Facultad de Arte.

ALCALÁ, JOSÉ RAMÓN, 2011. La Piel de la Imagen. Ensayos sobre gráfica en la cultura digital. Valencia: Sendemà Editorial.

ARTIAGA, JOSÉ. M; CAPILLA, PASCUAL; PUJOL, JAUME. 2002. Tecnología del color. Valencia: Universitat de València.

BLANCO-MORENO PEREZ, J. FRANCISCO. 2014. Reconstrucción de pintura mural mediante impresión directa con sistemas inkjet robotizados. Valencia: Universidad Politécnica de Valencia.

BREA, J. LUIS, 2005. Estudios visuales. La epistemologia de la visualidad en la era de la globalización. Madrid: Ediciones Akal.

BREA, J. LUIS, 2010. Las tres eras de la imagen. Imagen-Materia, Film, E-Image. Madrid: Ediciones Akal.

BOURRIAUD, NICOLAS, 2009. Radicante. Buenos Aires: Adriana Hidalgo editora. 
CASTRO, KAKO, [et al.], 2004. Inter (medios). La matriz intangible. Grupo de Investigación dx5. Facultad de Bellas Artes de Pontevedra: Universidad de Vigo.

CASTRO, KAKO, [et al.], 2006. Impresión piezoeléctrica. La estampa inyectada. Algunas reflexiones en torno a la gráfica digital. Grupo de Investigación dx5. Facultad de Bellas Artes de Pontevedra: Universidad de Vigo.

GALLARDO, VANESSA, 2015. La estampa que habita el espacio. Arte múltiple e instalación. Recorriendo los límites de la gráfica contemporánea. Valencia: Universidad Politécnica de Valencia.

GUBERN, ROMÁN, 1996. Del bisonte a la realidad virtual. La escena y el laberinto. Barcelona: Editorial Anagrama.

HOCKNEY, DAVID, 1994. Así lo veo yo. Madrid: Ediciones Siruela.

HWM, The Science of Inkjet Printing. 2001. № 51. ISSN 0219-5607.

MARTIN JÜRGENS, 2009. The digital print: Identification and preservation. Getty Conservation Institute.

PASTOR, JESÚS; ALCALÁ, JOSÉ R. 1997. Procedimientos de transferencia para la creación artística. Pontevedra: Dip. Prov. De Pontevedra.

RAUSCHEMBERG, ROBERT, 1998 - 1999. Retrospectiva. Catálogo de la exposición en el Museo Guggenhein Bilbao.

TORTOSA CUESTA, RUBÉN, 2004. Laboratorio de una mirada: Procesos de creación a través de tecnologías electrográficas. Valencia: Universidad Politécnica de Valencia.

Museo Internacional de Electrografía

http://www.mide.uclm.es/

Hard Disk Museum

http://harddiskmuseum.com/

Grupo dx5 - Digital \& Graphic Art Research de la Universidad de Vigo

http://grupodx5.webs.uvigo.es/

Solimán López

http://solimanlopez.com/

Rubén Tortosa

http://www.rubentortosa.com/ 